\title{
AMÉNAGEMENT DES PASSES A SAUMONS sur le LEGUER au barrage de KERNANSQUILLEC en PLOUVENEZ-MOËDEC (Côtes-du-Nord)
}

\author{
par A. GARDEBOIS
}

Architecte Honoraire

Les fleuves côtiers bretons sont, pour la plupart, plus ou moins remontés par les saumons et ont été, dès l'avant-guerre, pourvus d'ouvrages facilitant leur migration. Toutefois le Leguer, petit fleuve des Côtes-du-Nord, se trouvait défavorisé par l'existence, à $35 \mathrm{~km}$ de son estuaire, d'un important barrage. Cependant, il figure sur la nomenclature des rivières à saumons et est évidemment classé en $1^{\text {re }}$ catégorie.

II prend sa source entre BOURBRIAC et BULAT-PESTIVIEN à 240 mètres d'altitude au lieudit Penleguer (qui signifie Source du Guer) et court sensiblement du Sud au Nord sur un plateau de gneiss et de schiste au relief assez tourmenté. Ayant reçu de nombreux affluents, il atteint BELLE-ISLE-ENTERRE après avoir traversè la Forêt domaniale de COAT-AN-HAY. C'est à la sortie de ce bourg, dans une vallée élargie, qu'il reçoit son affluent principal le Guic, à 95 mètres d'altitude.

A $5 \mathrm{~km}$ en aval, il est arrêté par le barrage de KERNANSQUILLEC, haut de $16,35 \mathrm{~m}$, qui crée une retenue de $3 \mathrm{~km}$ de long.

Le Leguer est alors fortement encaissé dans les garnits où seules quelques routes le traversent, réunissant les plateaux qui le dominent. Elles facilitent aux pêcheurs l'accès du fleuve. D'autres petits ruisseaux, plus ou moins abondants, viennent le renforcer rive droite et rive gauche, et, après avoir sauté un certain nombre de barrages de moulins désaffectés de médiocre hauteur et facilement franchissables par les migrateurs, il atteint LANNION où se trouve, au Pont Sainte-Anne, la limite de salure des eaux, la limite de l'inscription maritime ètant au Moulin de KERIEL à $5 \mathrm{~km}$ en amont de LANNION.

S'infléchissant à l'Ouest dans un large estuaire où il serpente à marée basse mais qui se remplit avec le flot, il se jette dans la Manche (PI. 1), près de LOCQUEMEAU. 
La longueur de ce fleuve est de $70 \mathrm{~km}$ environ et sa pente moyenne assez prononcée, surtout dans la partie supérieure de son cours, est de 5 pour mille, soit 7 pour mille de la source à BELLE-ISLE-EN-TERRE et 3 pour mille en aval jusqu'à l'embouchure.

Comme nous l'avons dit, à $20 \mathrm{~km}$ de sa source à la sortie du bourg de BELLE-ISLE-EN-TERRE, il reçoit rive gauche le Guic qui nait à 250 mètres d'altitude près de GUERLESQUIN, à l'extrémité Est des Monts d'Arrée dans le Finistère dont il forme limite avec les Côtes-du-Nord; sa longueur est de $40 \mathrm{~km}$ environ et sa pente moyenne de 4 pour mille. II reçoit lui-même, à sa droite, le ruisseau de Saint-Emilion qui alimente sur son cours le bel étang de BEFFOU, à proximité de la Forêt domaniale du même nom, ainsi que le Loup.

Ces cours d'eau et leurs nombreux émissaires coulent Sud-Nord puis Ouest-Est, sur un plateau mollement ondulé et assez aride. Ils présentent ainsi que le Leguer, quoique à un degré moindre pour ce dernier, de nombreuses frayères : le débit du Guic à son confluent étant notablement plus important que celui du Leguer et son cours étant également plus attractif. TERRE :

C'est ainsi que les remontées s'effectuent en amont de BELLE-ISLE-en-

sur le Leguer iusqu'à la R.N. 787 de CALLAC à GUIMGAMP,

sur le Guic jusqu'à la R.D. 15 de LOQUIVY-PLOUGRAS à PLOUVENEZ-MOEDEC. sur le Loup jusqu'au lieudit SCALOU dans la commune de PLOUGONVER.

Aucune pollution appréciable ne vient contaminer les eaux de ce bassin dont le régime, fonction des pluies qui s'abattent sur la Bretagne, est assez. irrégulier; la végétation et la faune aquatiques y sont très riches et favorables.

La chute du barrage de KERNANSQUILLEC de 10,80 $\mathrm{m}$ alimente 3 turbines Francis de 600,300 et $175 \mathrm{CV}$ dont le courant était destiné à la Papeterie de BELLE-ISLE-en-TERRE appartenant à la Société Anonyme des Papeteries VALLEE. L'usine est aujourd'hui fermée et le courant provenant des alternateurs est distribué par le réseau de l'Electricité de France.

Ce barrage à parois minces et à voûtes multiples a été édifié en 1923 ; c'est le premier ouvrage en France. de ce type (PL. 2).

II est constitué par 14 voûtes en béton armé inclinées vers l'aval à $45^{\circ}$ et retenues par des contreforts. Ces voûtes vues sur un plan horizontal sont demi-circulaires de $4,66 \mathrm{~m}$ de diamètre dans œuvre; elles retiennent l'eau d'une part, et, d'autre part, cinq d'entre elles abritent les machines, un mur de façade étant élevé entre 6 des contreforts aval. Ceux-ci sont inclinés à $60^{\circ}$ sur l'horizontale, voûtes et contreforts entretoisés à la partie haute par une passerelle en béton armé rectiligne, longue de 70 mètres environ, qui parcourt la crête de l'ouvrage à $1,50 \mathrm{~m}$ au-dessus de la retenue dont le niveau légal est de $13,27 \mathrm{~m}$ (arbitraire).

Ce barrage comporte, sur sa rive gauche, un canal déversoir de 140 mètres de long, entaillé dans le granit et qui était destiné à constituer une passe à poissons naturelle. Son débouché, bien que situé à 100 mètres à l'aval des 3 turbines serait, si son débit était constamment suffisant, relativement attractif. En conséquence, les saumons auraient été susceptibles d'y remonter 


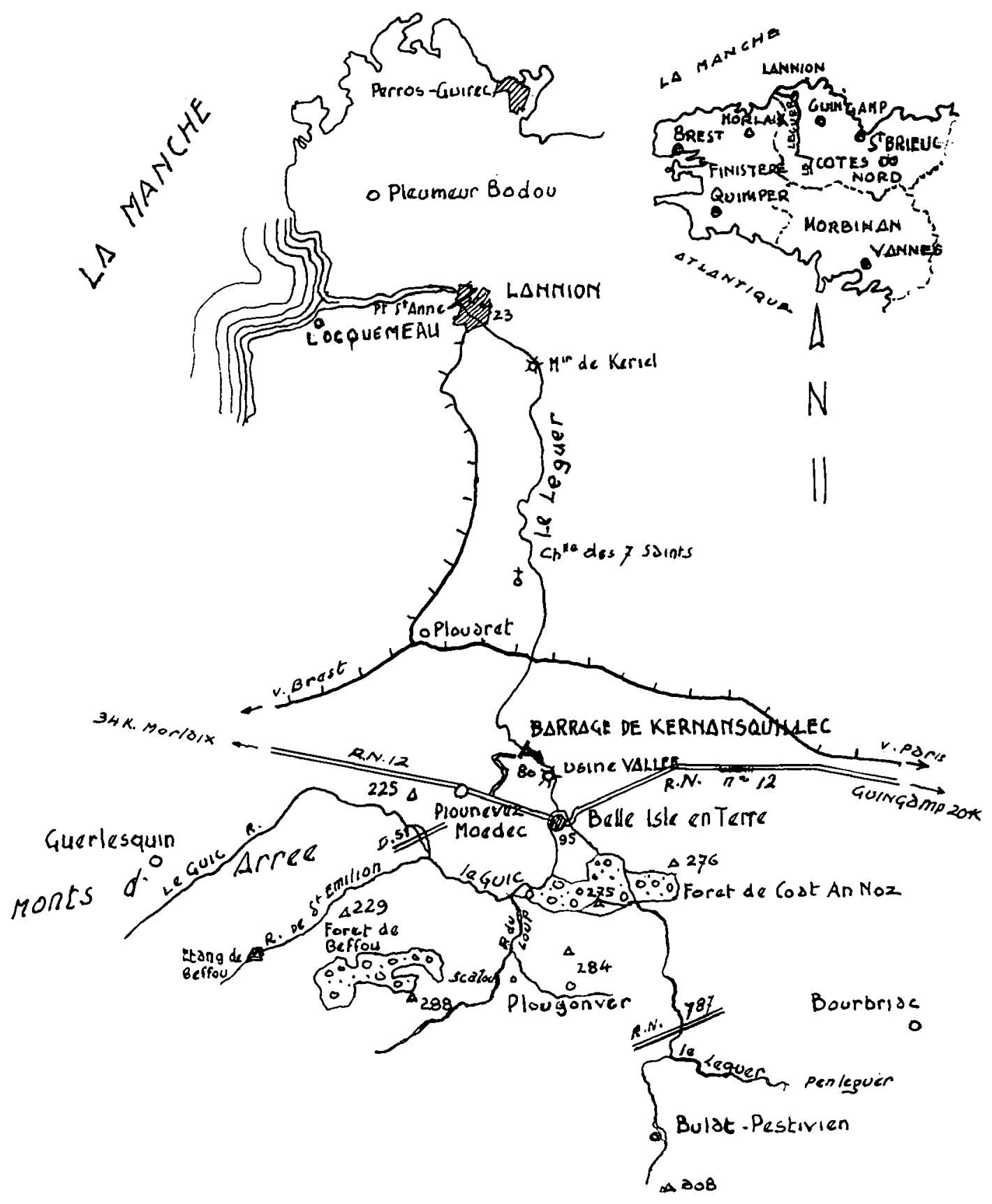

Planche 1 - Bassin du Leguer

Echelle 1/285000e 
si sa partie amont barrée par les 3 vannes déversoirs du barrage avait été aménagée dans ce but.

En 1952, le Conseil supérieur de la Pêche ayant décidé de faciliter le franchissement des obstacles qui s'opposent à la remontée des saumons dans le Leguer, subventionna la Fédération des Associations de Pêche et de Pisciculture des Côtes-du-Nord en vue de la construction d'ouvrages facilitant cette remontée.

A vrai dire, cet aménagement se fit en trois étapes.

Monsieur l'Ingénieur des Eaux et Forêts BACHELIER, dont l'expérience en ce domaine nous fut précieuse, et nous-même étudiâmes d'abord les différentes solutions possibles, étant précisé que le propriétaire de l'ouvrage, Monsieur VALLEE, accordait à la Fédération toutes facilités possibles à condition... qu'il ne soit pas touché au barrage.

Cette interdiction écartait la création d'une écluse ainsi que d'une échelle à amortisseurs qui, toutes deux, auraient entaillé la crête de l'ouvrage audessous du niveau légal.

La seule solution possible était donc, si nous pouvons dire, "d'enjambers le barrage avec un ascenseur dont le pied se trouverait dans le canal de fuite de la turbine la plus puissante côté rive droite et qui, pour réduire la charge à élever et l'énergie consommée (fournie gratuitement par l'usine), remonterait les poissons à sec au-dessus de la crête de l'ouvrage, assez vite pour qu'ils ne souffrent pas de ce transport aérien, et les ferait glisser dans le bief supérieur.

\section{1 - ASCENSEUR \\ - DESCRIPTION -}

Cet ascenseur se compose d'un carrelet métallique reposant sur le radier du carial de fuite de la grosse turbine de $600 \mathrm{CV}$ et qui, à la sortie de son conduit d'aspiration, s'élève périodiquement dans une cage en charpente métallique traditionnelle, à l'aval du barrage, au moyen d'un treuil mû électriquement. La hauteur à franchir est de 17,64 $\mathrm{m}$ dont 15,28 $\mathrm{m}$ hors de l'eau (PL. 3).

A la partie haute, ce carrelet déverse son contenu dans une goulotte en bois qui plonge dans le bief supérieur du barrage. II redescend aussitôt pour reprendre sa position de capture au fond du radier.

A - CARRELET. - Ce carrelet est constitué par une cage métallique parallélipipédique de $2.82 \times 1.744$ au fond et dont les côtés verticaux ont $1,50 \mathrm{~m}$ de hauteur. (PL. 4).

Les deux petits côtés latéraux de $1,744 \mathrm{~m}$ de long parallèles aux contreforts du barrage, sont maintenus verticalement par soudure au cadre du fond.

Les deux grands côtés perpendiculaires au sens du courant ont cette même hauteur, le côté amont étant fixe, le côté aval étant mobile autour du grand côté du cadre formant fond du carrelet.

C'est à ces grands côtés, dont l'un est mobile, que sont accrochés les 4 brins rigides fixés à une chaine Gall de relevage.

Ainsi, quand le fond du carrelet se pose sur le radier du canal de fuite 


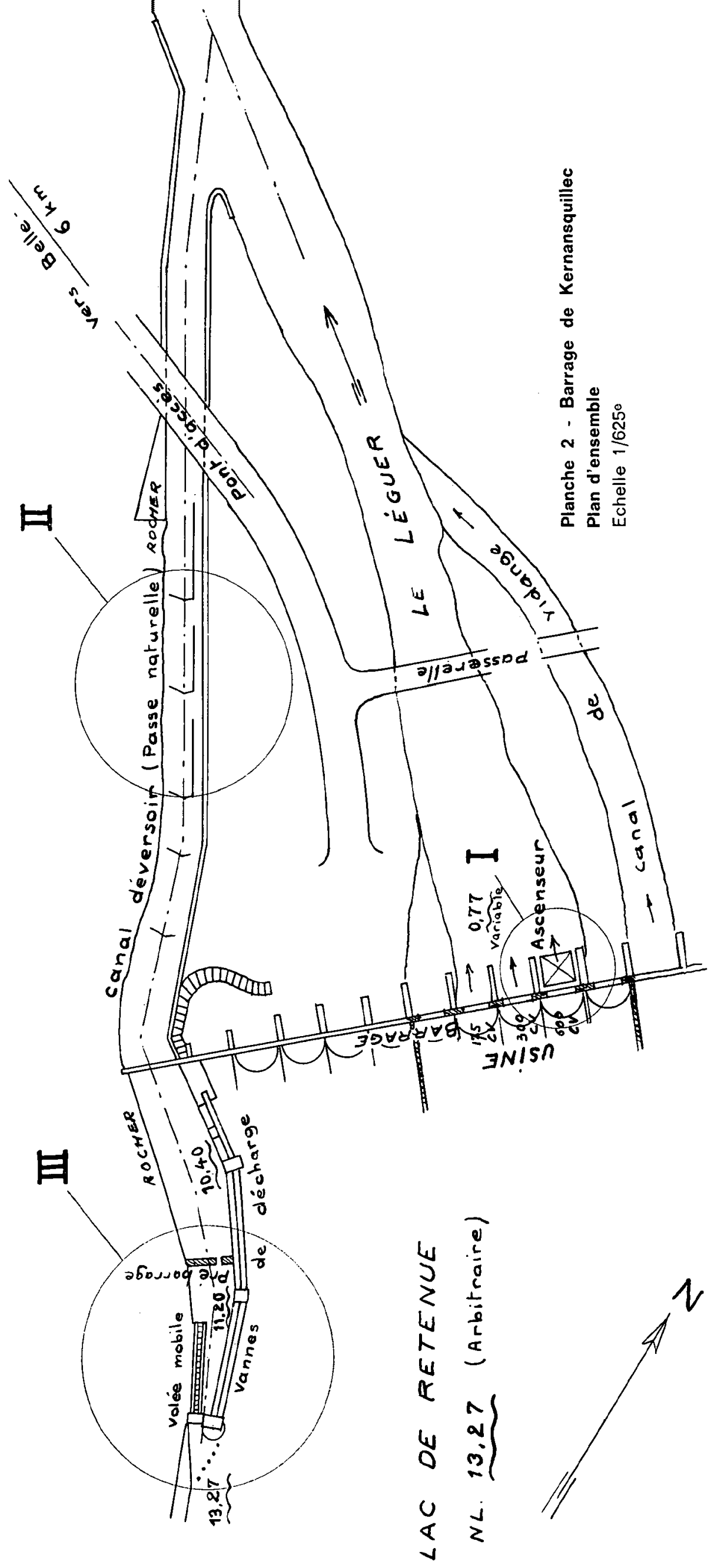




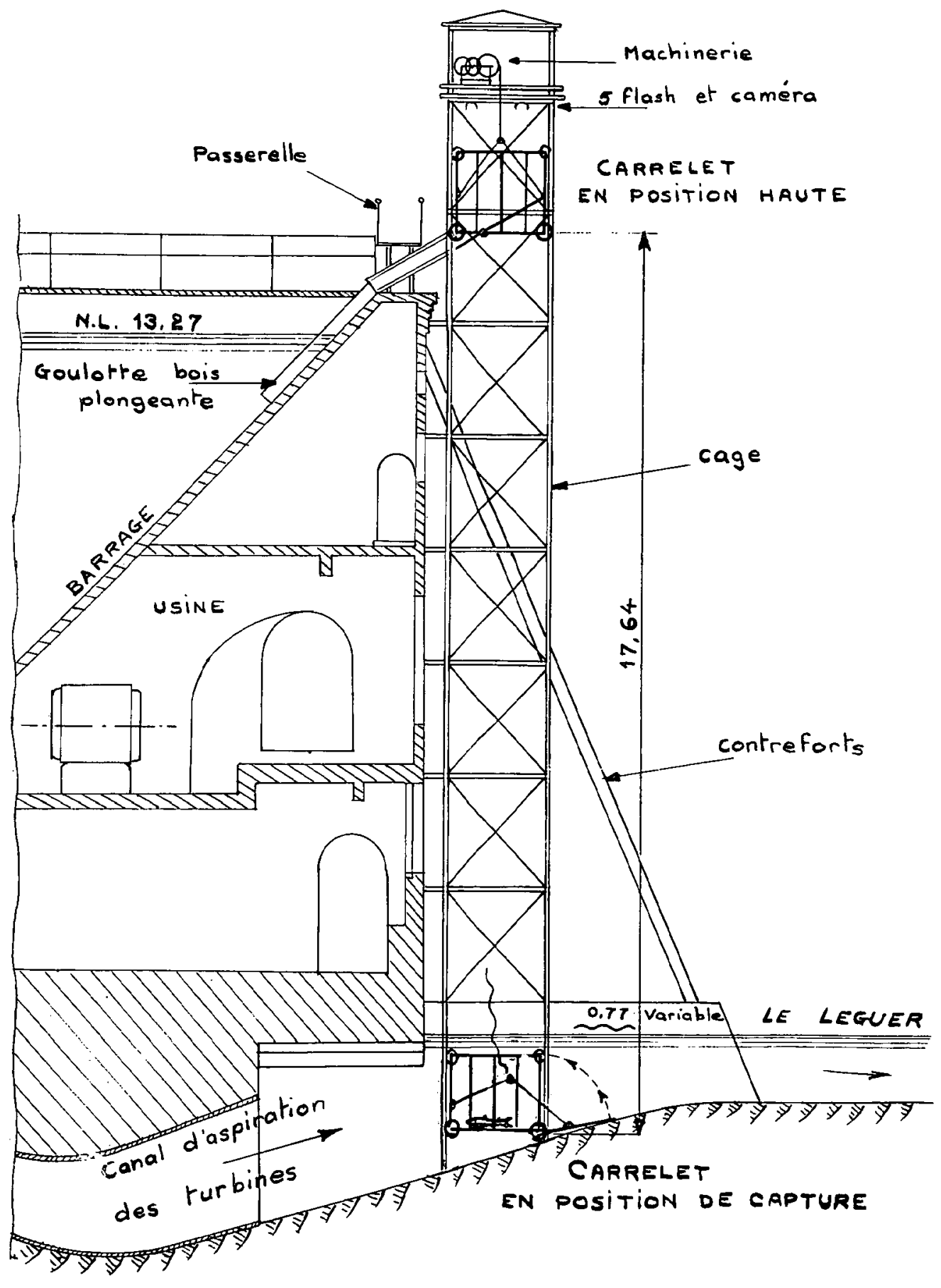

Planche 3 - Ascenseur (coupe) Echelle 1/125e 
de la turbine, le côté aval transversal s'y rabat pour former une surface de capture de $2.83 \times(1.465+1.744)=9 \mathrm{mq} 05$ de surface.

Le fond du carrelet peut osciller autour d'un axe solidaire du cadre du fond et parallèle aux grands côtés de celuic-i, à 0.546 du côté amont et à 1.146 du côté aval (P.L. 5).

Les 5 panneaux du carrelet ( 1 fond et 4 côtés) sont constitués par des fers $1 / 2$ rond creux de $15 \mathrm{~mm}$, espacés de $50 \mathrm{~mm}$, soudés sur les cadres en tube acier de $26 / 34$ et sont protégés contre l'oxydation.

Toutefois, le côté aval du cadre du fond oscillant est en fer plein rond de $50 \mathrm{~mm}$ pour former contrepoids et assurer ainsi la fermeture en position horizontale du fond oscillant.

Le côté amont du cadre du fond oscillant reçoit 2 crochets qui, quand le carrelet est remonté et atteint le niveau de la goulotte de déversement, y buttent et forcent le fond oscillant à s'incliner et à "bailler " avec une pente maximum de $30 \circ$ vers ladite goulotte à l'amont.

Le guidage du carrelet dans la cage en charpente métallique est assuré par 4 galets en bronze et sa course est limitée aux positions inférieure et supérieure par des butoirs.

B - CHARPENTE. - La cage de charpente métallique dans laquelle s'élève le carrelet est composée de 4 montants en fer en $U$ formant guides dans lesquels roulent les galeis en bronze du carrelet.

Les montants sont entretoisés en fers cornières avec des liens formant Croix de St André, le tout assemblé avec gousset en tôle et boulons.

A la partie basse, ils sont fixés dans 2 traverses scellées dans les contre. forts, les fixations dans le radier du canal de fuite ayant été irrèalisables.

Latéralement, ils sont maintenus par des scellements dans les contre. forts. et sur la façade aval verticale de la salle des turbines. Cette cage s'élève au-dessus de la passerelle couronnant le barrage. Elle est haubannée à la partie haute pour résister aux efforts du vent et close sur ses 4 faces avec couverture à deux pentes pour abriter l'appareillage.

C - GOULOTTE. - La goulotte destinée à recevoir les poissons à la partie haute de la cage est réalisée en frêne et en chêne.

Formant entonnoir rectangulaire de 3 mètres de large contre la cage, inclinée à $45 \circ$, elle se rétrécit progressivement pour mesurer 1 mètre de large sur 0,20 de haut à la base.

Close sur toutes faces, son orifice inférieur ouvert plonge de $1,50 \mathrm{~m}$ audessous du niveau légal dans le bief amont afin d'éviter le braconnage.

Une passerelle métallique eniambe la goulotte et permet la circulation et l'accès à l'appareillage : moteur, treuil, caméra, etc...

D - MOTEUR. - Le moteur électrique est placé sur un plancher à la partie supérieure de la cage; il est commandé par une minuterie; sur son axe est calé le treuil d'enroulement de la chaine Gall de relevage. Moteur et treuil sont dans un carter ètanche et isolé.

Des contrepoids équilibrent le carrelet. 

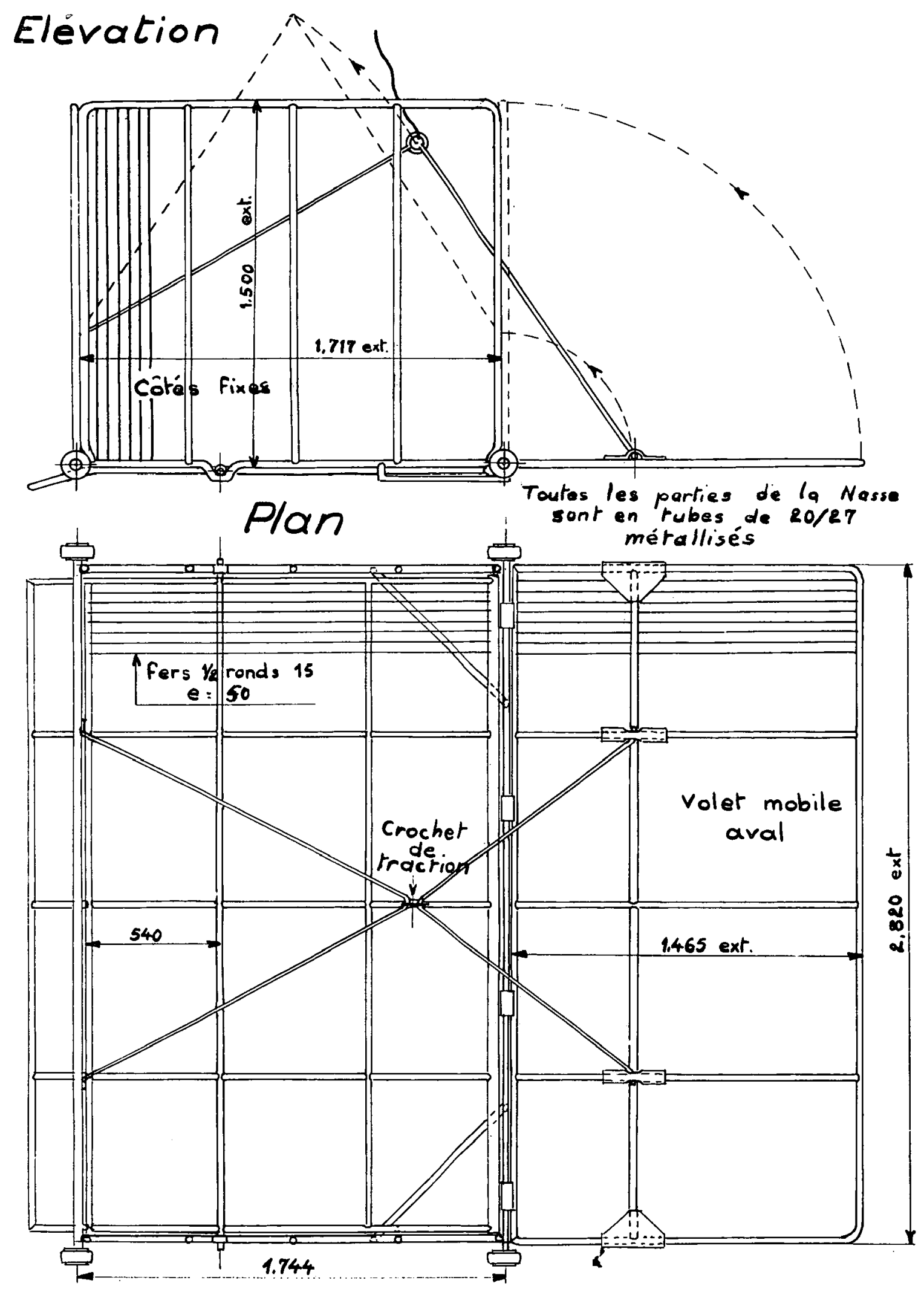

Planche 4 - Carrelet (détail)

Echelle 1,25e 


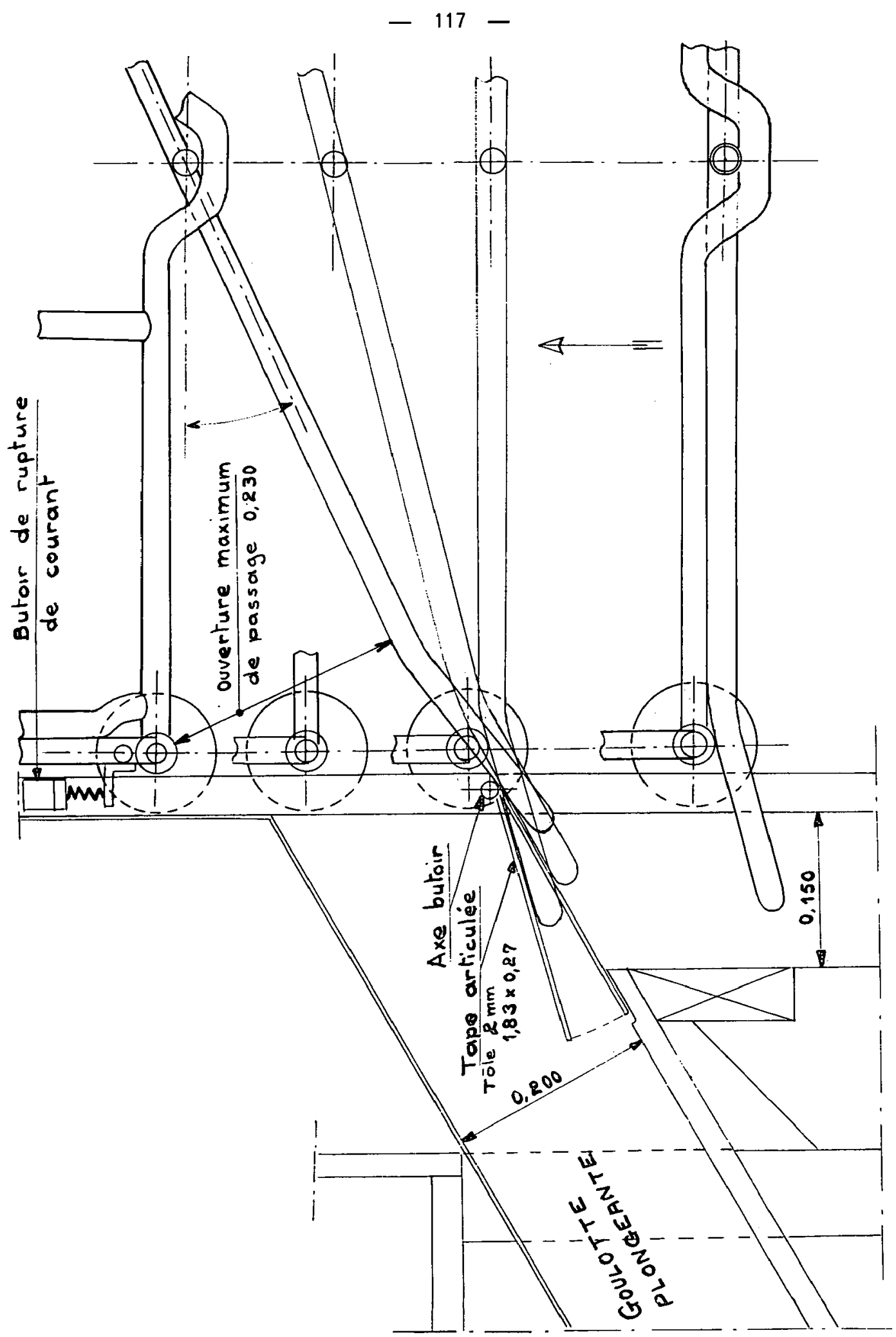

Planche 5 - Carrelet en position haute

Echelle $1 / 6,25^{\circ}$ 
Braquée vers le bas, une caméra a été placée, sur l'initiative de Monsieur l'Ingénieur principal BACHELIER en haut de la cage d'ascenseur sous le plancher du moteur et du treuil; 5 flashes commandés par le carrelet en position haute éclairent ledit carrelet qui est ainsi photographié avec son éventuel contenu avant chaque déversement.

A l'intérieur de la salle des turbines, la minuterie commande un inverseur de courant qui permet l'enroulement du câble de la montée ou son déroulement à la descente, avec l'appareillage de sécurité nécessaire.

\section{FONCTIONNEMENT}

En position de capture, le carrelet, ainsi que nous l'avons dit, repose sur le radier du canal de fuite de la turbine (PL. 3).

Les 3 volets latéraux, perpendiculaires au fond du carrelet sont verticaux et fixes; ils empêchent les poissons de s'engager dans le conduit d'aspiration de la turbine.

Le volet mobile aval n'étant pas retenu par le câble de traction qui présente un certain \& mou ", s'est rabattu sur le radier, entraîné par son poids. La surface de capture est donc de $9,05 \mathrm{mq}$.

Quand la minuterie, à l'expiration d'un temps déterminé (30 minutes), met en marche le moteur, le treuil enroule la chaine Gall à la vitesse de remontée de $0,50 \mathrm{~m}$ seconde.

Les 4 brins attachés, à raison de 2 sur le volet amont et 2 sur le volet aval, commencent par relever ce dernier lequel s'oppose à la fuite des poissons capturés.

A ce moment, le carrelet constitue une caisse à claire-voie de $2.82 \times 1.744$ et $1,50 \mathrm{~m}$ de hauteur.

Les volets relevés entrainent le fond du carrelet dans son mouvement ascensionnel, les saumons étant remontés à sec pendant les 30 secondes que dure cette élévation.

Arrivé à la partie haute au niveau de la goulotte amont, cette dernière accroche le fond oscillant du carrelet, alors qu'il continue à monter de $10 \mathrm{~cm}$. C'est à ce moment que les flashes éclairent et que la caméra enregistre.

Le fond oscillant prend alors une inclinaison. qui atteint 300, ménageant une ouverture de $23 \mathrm{c} / \mathrm{m}$ entre la base du volet fixe amont et le cadre du fond.

Les poissons entrainés par la pente s'engagent par la tape en tôle dans la goulotte en bois, à l'intérieur de laquelle ils glissent vers le bief amont.

Quelques instants après, le moteur commandé par la minuterie tourne en sens inverse, le carrelet descend, le fond oscillant liberé reprend sa position horizontale maintenue par le fer rond plein de $50 \mathrm{~mm}$ formant contre-poids.

Arrivé sur le radier du canal de fuite, il s'y repose, les brins cessant de tirer sur le volet aval mobile, ce dernier s'aplatit sur le radier, en position de capture, prêt à recommancer un nouveau cycle. 


\section{II - amelioration du CANAL DeVersoir rive gauche}

en vue de son utilisation comme échelle à saumons

Le canal déversoir de la retenue, recevant l'ècoulement des vannes secteur en période de crue est lang de 140 mètres environ et large de 4 à 5 mètres; il a été creusé lors de la construction, du barrage dans la rive gauche du Leguer avec l'espoir qu'il formerait une passe naturelle pour les saumons, sa pente moyenne étant de $10 \%$ (PL. 6).

En fait, il n'est alimenté qu'aux périodes de crue quand les turbines sont incapables d'absorber le débit de la rivière ou lors du chômage hebdomadaire de l'usine et sa disposition ne permettait que de rares remontées de migrateurs.

Ceux-ci arrivaient parfois au niveau de barrage au droit des vannes déversoirs, mais ils devaient encore franchir une diffèrence de niveau de 3 mètres. Or, le bassin intermédiaire créé il y a quelques années à cet endroit, ne facilitait nullement le franchissement de cet obstacle.

En conséquence, en 1955 dans le but de favoriser la remontée des saumons, il a été exécuté, entre le pont à l'aval du canal déversoir et la partie amont du dit canal, une série d'ouvrages destinés en période de crue à ralentir la vitesse du courant sur une certaine portion du canal et, en période de basses eaux, à réserver un débit suffisant avec l'épaisseur d'eau nécessaire sur une partie de la largeur du canal formant ainsi une véritable échelle.

Les ouvrages sont constitués par des bajoyers en béton armé, parallèles à la rive, encastrés dans le radier du canal et laissant un passage de 1 mètre côté rive droite.

Des amortisseurs de LACHADENEDE en tôle, espacés de $0,75 \mathrm{~cm}$ environ, ont été scellés dans le couloir ainsi créé.

De place en place, des murets en béton sensiblement perpendiculaires au courant dirigent le flot vers le couloir latéral.

A la partie amont au droit des vannes déversoirs, une volée d'échelle à saumons en béton armé munie d'amortisseurs de LACHADENEDE et de 1 mètre de large fut construite pour compenser la différence de niveau; slle avait 14 mètres de long et une pente de $15 \%$.

Une vanne à guillotine en chêne condamnait l'ouvrage, la pelle étant arasée à la partie supérieure au niveau légal de retenue 13,27 (arbitraire).

Cet aménagement a permis à un certain nombre de migrateurs particulierement vigoureux d'arriver au pied des vannes déversoirs du barrage mais ils étaient incapables d'aller plus loin, le couloir de l'échelle étant rarement alimenté par suite des variations de niveau de la retenue, elle-même fonction du débit du Leguer et de l'énergie absorbée par les turbines.

Par ailleurs, l'expérience a montré que les saumons ne remontaient que par un débit de 250 à 300 litres seconde dans le chenal, au-delà de ce régime l'eau est trop émulsionnée. 


\section{III. - VOLEE MOBILE}

\section{à la partie haute du canal déversoir formant échelle à saumons.}

En raison de l'exploitation du barrage par éclusées d'une amplitude de 1,75 à 2 mètres, la volée amont de l'échelle de LACHEDENEDE n'était pour ainsi dire jamais alimentée (PI. 7). Mais les utilisateurs du barrage devant réserver dans le canal déversoir un débit de 300 litres seconde, ce débit réservé crée un appel dans le Leguer sur la rive gauche à 100 mètres environ à l'aval du barrage. déversoir.

En conséquence, des remontées partielles se faisaient par ce canal

C'est ainsi qu'au cours de la période du 22 mars - 26 mai 1964, 21 saumons ont été vus dans un pool, à l'amont du pont, à 40 mètres environ de l'origine aval du canal déversoir.

Deux saumons seulement ont atteint le pied de la dernière partie de l'échelle à l'amont qu'ils n'ont pu franchir, celle-ci n'étant pas alimentée par suite des éclusées de l'usine.

Ces 21 saumons ont été capturés par les gardes fédéraux et immergés dans la retenue.

Lors de notre tournée du 21 juillet 1965, nous avons vu à la partie haute, au pied des vannes déversoirs et de la dernière volée d'échelle, à sec, une quinzaine de belles truites "fario et une truite de mer.

II était donc nécessaire d'améliorer cette passe naturelle, de trouver le débit optimum devant l'alimenter, les saumons étant arrêtés aussi bien par un débit trop faible que par une alimentation excessive, comme il a été dit ci-dessus.

Quoiqu'il en soit, d'après les engagements pris dans le règlement d'eau, il est possible de compter sur un débit réservé de 300 litres seconde environ, quel que soit le niveau amont de la retenue.

En 1964, Monsieur le Docteur AURAGAN, Président de la Fédération des A.P.P. des Côtes-du-Nord, nous demanda d'étudier un dispositif permettant aux migrateurs de franchir ce dernier obstacle, compte tenu des variations de niveau à l'amont.

Seule une passe mobile réunissait ces conditions : un appareil de ce type avait été employé 15 ans auparavant au Bec-des-Deux-Eaux sur la Vienne.

Cette passe mobile ou échelle mobile a été axée à l'emplacement de l'ancienne échelle de LACHADENEDE dont les bajoyers ont été surélevés en béton de ciment aux cotes prévues, la largeur du couloir étant ramenée à 0,60 . Deux massifs également en béton prennent appui, rive droite sur la culée amont de la vanne secteur formant déversoir d'extrémité et, rive gauche, sur les rochers de la berge; ils calent le flotteur de la volée d'échelle mobile qui est guidé par des coulisses en orme.

La course de cette dernière varie entre $13,27 \mathrm{~m}$ niveau légal et $11,80 \mathrm{~m}$, soit 1,47 m. Elle absorbe, en partie, les éclusées du barrage.

Cette volée mobile est constituée par une goulotte de section rectangulaire de $0,50 \mathrm{~cm}$ au fond avec deux bajoyers verticaux en tôle de $4 \mathrm{~mm}$ d'épaisseur 
Planche 6 :

Canal déversoir

Aménagement de la passe naturelle

Echelle 1!250e 
Planche 7 :

Volée mobile à l'extrémité

amont du canal déversoir

Echelle $1 / 62,5 \mathrm{e}$ 


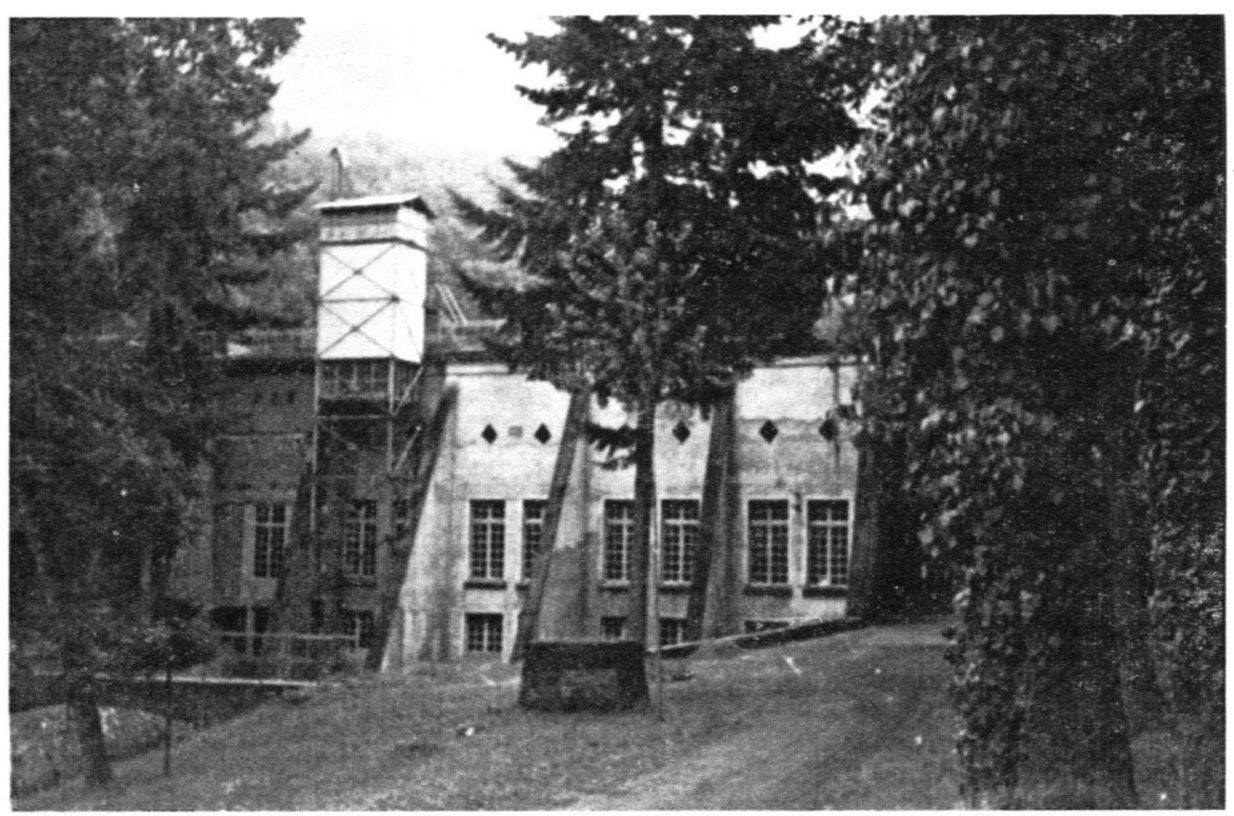

Ascenseur de Kernansquillec (cliché Biver)

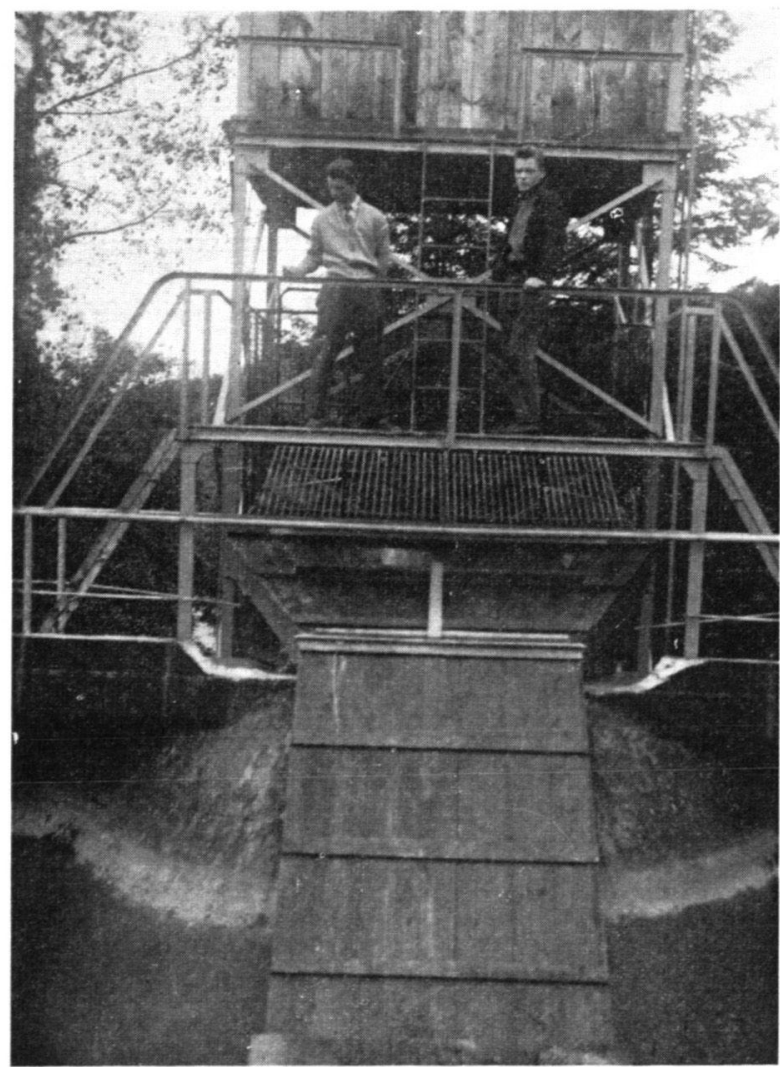

Carrelet en position haute et goulotte 
et $0,50 \mathrm{~cm}$ de haut, fixés sur deux longrines en $L$ de 160 entretoisées et réunies par un platelage en chêne rainé de $54 \mathrm{~mm}$ d'épaisseur.

La longueur de cette échelle mobile, prise de son axe de pivotement inférieur en bronze à son extrémité, est de $10,50 \mathrm{~m}$.

Un flotteur en tôle d'une poussée de $1000 \mathrm{~kg}$ fait corps avec la partie amont de cette volée; il s'évase en entonnoir pour faciliter la descente des tacons et doit être lesté pour obtenir la lame d'eau prévue.

Avec la pente maximum de la volée qui est de $167 \mathrm{~mm}$ par mètre correspondant au niveau légal de $13,27 \mathrm{~m}$, son enfoncement réserve une lame d'eau de $0,30 \mathrm{~cm}$ qui donne justement un débit de 300 litres seconde correspondant au débit réservé avec une vitesse de courant de 3,50 mètres seconde.

La pente minimum de $20 \mathrm{~mm}$ par mètre correspond à un niveau d'étiage de $11,80 \mathrm{~m}$ qui, en recevant également une lame d'eau de $0,30 \mathrm{~m}$, donne un débit de 225 litres seconde, avec une vitesse de courant de $1,50 \mathrm{~m}$.

Des butées haute et basse limitent la course de l'appareil.

L'expérience a montré que quelques réducteurs de vitesse ètaient nécessaires; ils furent constitués par des cales en chêne, clouées sur le platelage du fond également en chêne et espacées d'un mètre.

Une vanne en bois à guillotine est fixée à l'amont pour condamner l'ouvrage en cas de nécessité avec emplacement pour double batardeau et un portique de relevage du flotteur est établi pour soulever éventuellement l'appareil.

Afin d'éviter l'accumulation des détritus divers passant au travers des grilles de protection et qui viendraient coincer l'appareil, un voile en caoutchouc très robuste se replie sous ce dernier et une buse de $20 \mathrm{~cm}$ de diamètre forme, en outre, vidange de la cuvette sous les flotteurs. Elle peut être obturée.

Un dispositif d'étanchéité est prévu pour évier les pertes latérales, constitué par un bourrelet de caoutchouc fixé sur chaque face en tôle et frottant sur les 2 parois de la maçonnerie de l'ouvrage.

Un barrage de surface, avec barreaux verticaux fixes, très résistants, est établi à l'amont de la volée jour éviter l'introduction de corps flottants parfois volumineux en période de crue, tels que troncs d'arbres ou autres épaves flottantes.

L'aménagement de l'accès au bassin de repos, à la base de la passe mobile, a été exécuté à la demande après mise en eau de ladite passe afin d'examiner la direction des courants.

II est constitué par un pré-barrage créant une profondeur d'eau de 1 mètre environ au pied de la passe mobile à 6 mètres de celle-ci avec un déversoir échancré de $0,60 \mathrm{~m}$ de large, débitant lui aussi 300 litres seconde.

\section{CONCLUSION}

ASCENSEUR - Quelques années après la mise en service de cet appareil, les résultats furent encourageants, ceux-ci étant contrôlés de visu par l'électricien de service aux turbines et aux alternateurs, chaque fois que l'appareil 
s'élève, et, d'autre part, par la caméra qui a enregistré d'excellents clichés, étant précisé que les remontées de saumons se font de mars à novembre, la fraie ayant lieu en novembre ei décembre.

Ces remontées, ainsi que le montrera le tableau ci-dessous, sont très irrégulières et fonction du régime des eaux ainsi que de la marche des turbines, les saumons étant appelés par une crue et se déplaçant à ce moment. En conséquence, les premiers qui se présentent au pied de l'ascenseur ont séjourné l'été dans la partie basse du fleuve, alors que les poissons plus tardifs viennent directement de la mer.

Remontées constatées dans l'ascenseur de 1958 à 1967

\begin{tabular}{||l|c|c|c|c|c|c|c|c|c|c||}
\hline \multirow{2}{*}{ Février } & 1958 & 1959 & 1960 & 1961 & 1962 & 1963 & 1964 & 1965 & 1966 & 1967 \\
\cline { 2 - 4 } & & & 1 & & & & & & & \\
Avril & & 1 & & & 3 & 6 & 3 & 4 & 1 \\
Mai & & & 3 & & 5 & 36 & 1 & 30 & 5 \\
Juin & & 8 & 1 & & 13 & 12 & 17 & 32 & 25 \\
Juillet & & & & & & 5 & 10 & 2 & 12 & 5 \\
Août & & & & & & & & & 2 & 2 \\
Septembre & & & & & & 1 & & 1 & & \\
Octobre & & & 30 & & & & 6 & 3 & 31 & 22 \\
Novembre & & & 7 & & & 11 & & 21 & 33 & 18 \\
Total annuel & 23 & 6 & 47 & 4 & 12 & 39 & 70 & 55 & 144 & 103 \\
\hline
\end{tabular}

NOTA. - Dans le tableau ci-dessus, il n'a pu être fait de ventilation mensuelle pour les années 1958, 1959 et 1962.

En outre le fonctionnement de l'ascenseur a eu quelques défaillances au cours des étés de 1963,1964, 1965 par suite du mauvais état du carrelet qui a été remplacé en 1966, ce qui a eu une répercussion sur les remontées.

Les totaux ci-dessus constituent un minimum, quelques poissons ayant pu échapper à la surveillance du personnel de l'usine, qui tend à se relâcher.

Cette progression est due en grande partie à la fraie des reproducteurs qui ont maintenant accès aux frayères naturelles, l'ascenseur ayant libéré plus de $60 \mathrm{~km}$ de ces dernières.

D'autre part, des œufs et des tacons ont été immergés dans les petits émissaires des cours supérieurs du Leguer et du Guic à quelques dizaines de mètres de ces derniers par les gardes fédéraux, suivant le tableau d'alevinage ci-dessous :

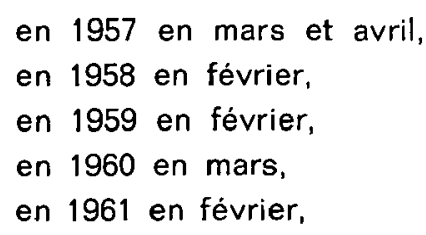

Alevinage constitué par un certain nombre de boites VIBERT garnies d'œufs fournis par le C.S.P. 
en 196453 boites VIBERT et 8000 alevins de saumons à vésicule résorbée,

en 196540 boites VIBERT,

en 196635 boites VIBERT et 3000 alevins de 3 mois,

en 196738 boites VIBERT et 205 tacons,

en 196855 boites VIBERT, 10000 alevins à vésicule résorbée et 2100 tacons de 8 à $18 \mathrm{~cm}$,

alevins et tacons provenant de la fécondation de reproducteurs saumons achetés aux inscrits maritimes produit de leur pêche sur le Trieux et élevés à la Salmoniculture fédérale de COAT-MEN.

En conséquence des opérations ci-dessus, en mai et juin 1965, la présence de très nombreux tacons fut constatée dans la retenue du barrage; ils profitèrent de l'ouverture des vannes de décharge pour dévaler.

En mai 1967 il en fut de même, des milliers de tacons étant observés dans le barrage qui dévalèrent quand les vannes furent levées et gagnèrent le Leguer par l'échelle aménagée dans le canal déversoir.

Le nombre des captures de saumons par les pêcheurs à l'aval du barrage (ni le Leguer, ni le Guic ne sont pêchés à l'amont du barrage) peut être évalué :

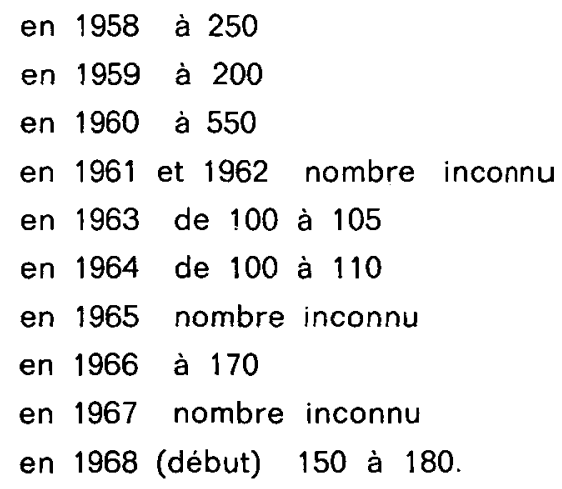
pêché :

Pendant une période à peu près semblable, les inscrits maritimes auraient

en 1958 de 700 à 800 saumons

en 1960 de 1200 à 1300 saumons

en 1964 de 100 à 120 saumons

en 1966 environ 300 saumons.

Aujourd'hui aucun inscrit ne pêche dans la partie maritime du Leguer à l'exception de 3 pêcheurs à pied.

Les résultats obtenus permettent donc de retenir ce type d'appareil pour équiper certains barrages existants de moyenne hauteur quand il est dangereux de les échancrer pour alimenter une passe ou une écluse, ou bien encore, quand il s'agit de barrages en matériaux meubles compactés qu'il serait téméraire de décompresser. Toutefois, la source d'énergie motrice ne devrait pas être éloignée afin d'éviter l'installation d'une ligne de transport de force onéreuse.

ECHELLE A SAUMONS et VOLEE MOBILE. - Là-encore de bons résultats furent obtenus car dès sa mise en eau le 9 août 1966, les truites remontaient le pré-barrage ; Le 10 août 1966, au soir, pendant le chômage de l'usine, deux sau- 
mons étaient vus dans le bassin de repos constitué au pied de la volée mobile; le lendemain, ils avaient franchi cet obstacle.

Le garde fédéral estime à 10 le nombre des saumons qui y sont passés du ler novembre 1967 au 31 décembre 1967, étant précisé que l'échelle n'est alimentée qu'en fin de semaine; de plus, de très nombreuses truites empruntent ce passage.

\section{NOTE TECHNIQUE \\ I. - ASCENSEUR (1953 - 1954)}

- ChARPENTE METALliQue. Elle fut exécutée par la Société métallurgique de Bretagne à SAINT-BRIEUC (22).

C'est un ouvrage de construction traditionnelle en fer à $U$, fers corniières, boulons et écrous, les grandes longueurs ne pouvaient être utilisées en raison des difficultés d'accès au chantier par des chemins étroits, sinueux et encaissés.

Le carrelet, comme il est décrit ci-dessus, pèse 300 kilogs environ.

\section{COUSIN, à TOURS (37).}

II comprend :

mouvement périodique constitué par une minuterie BRILLE réglable, de $0 \mathrm{~h} 30$ à $3 \mathrm{~h}$ maximum, déclanchant la montée du carrelet jusqu'au point de déchargement au sommet. Un disjoncteur est placé dans le bâtiment attenant, avec le dispositif horaire à temps. Tous les autres appareils sont fixés dans un coffre, près du groupe de levage.

Le groupe-treuil, avec réducteur à vis sans fin, moteur électrique de $2 \mathrm{CV}$ $1 / 2$ et frein électro-magnétique, assure l'arrêt exact, à + ou $-5 \mathrm{~cm}$; il est alimenté en 220 volts.

La montée s'effectue à $0,50 \mathrm{~m}$ seconde soit une durée totale du parcours de 36 secondes dont 30 secondes hors de l'eau.

Traction par. chaine Gall de résistance : $700 \mathrm{~kg}$ entrainée par pignon en acier spécial à laquelle sont suspendus le carrelet d'une part, et le contre-poids constitué par des rails équilibrant celui-ci d'autre part.

L'appareillage électrique, nécessaire au bon fonctionnement de cette installation : arrêt de fin de course, relais de démarrage d'inversion, d'automaticité et de sécurité, disioncteur, interrupteur horaire et bouton de commande à main, câble de l'installation du moteur ont été mis en place dans la salle des turbines.

- DIVERS : les scellements, la goulotte, la peinture furent exécutés par l'Entreprise LE GUILLERMIC à BELLE-ISLE-en-TERRE (22).

- CAMERA et flashes installés par Monsieur JOUBERT, photographe à SAINT-BRIEUC (22).

Le coût de cet ouvrage qui constitue un prototype s'est élevé en 1954 à la somme de 3.455.154 Anciens Francs (valeur 1968:68 000 Francs environ).

NOTA. - En 1966, après douze ans de service, le carrelet malgré sa protection, très oxydé par les eaux de Leguer dut être remplacé et nous en avons profité pour renforcer ses membrures. De même une révision complète de l'appareillage qui travaille dans des conditions climatiques très rigoureuses fut opérée. 

II - AMENAGEMENT de l'ECHELLE à SAUMONS dans le CANAL
DEVERSOIR (1955)

- Les ouvrages en béton, barrages, scellements, etc... furent exécutés par l'Entreprise LE GUILLERMIC à BELLE-ISLE-en-TERRE (22).

- Les amortisseurs de LACHADENEDE étant fournis par les Chantiers DUBIGEON, à BREST (29).

Le coût de cet ouvrage s'éleva en 1955 à 1.196.100 Anciens Francs, (valeur 1968 : 17000 Francs).

\section{III - CONSTRUCTION de la PARTIE d'ECHELLE MOBILE (1965-1966)}

Les travaux furent étalés sur 2 ans ne pouvant être exécutés qu'en août. mois de chômage des installations électriques, turbines et alternateurs.

- La partie mobile, les vannage et portique de relevage furent exécutés par la Société Métallurgique de Bretagne à ST-BRIEUC (22),

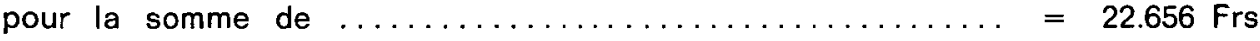

(là-encore les difficultés d'accès furent grandes)

Les maçonnerie, ouvrages en béton, enduits, barrage amont, fouilles qui exigèrent de grandes précautions avec un chantier d'approvisionnement extrêmement difficile furent menés à bien par l'Entreprise LE GUILLERMIC de BELLE-ISLE-en-TERRE (22) et coûtèrent $=44.678$ Frs

TOTAL ...... $=\overline{67.334 \text { Frs }}$

Juillet 1968 